Article

\title{
Chemical Space Charting of Different Parts of Inula nervosa Wall.: Upregulation of Expression of Nrf2 and Correlated Antioxidants Enzymes
}

\author{
Xiang-rong Cheng ${ }^{1,2,3, *(\mathbb{D})}$, Wei Zhao ${ }^{1}$, Wen-le Dong ${ }^{1}$ and Guo-wei Le ${ }^{1,2,3}$ \\ 1 School of Food Science and Technology, Jiangnan University, Wuxi 214122, China; \\ zhaowei1997w@163.com (W.Z.); dddddddddddd1212@163.com (W.-1.D.); lgw@jiangnan.edu.cn (G.-w.L.) \\ 2 National Engineering Research Center for Functional Food, Jiangnan University, Wuxi 214122, China \\ 3 Collaborative Innovation Center of Food Safety and Quality Control in Jiangsu Province, Jiangnan \\ University, Wuxi 214122, China \\ * Correspondence: cheng-xiangrong@hotmail.com; Tel./Fax: +86-0510-8591-7780
}

Received: 20 September 2020; Accepted: 13 October 2020; Published: 19 October 2020

\begin{abstract}
The edible and medicinal part of Inula nervosa Wall. (Xiaoheiyao) is confined to its root without sufficient phytochemical and biological investigation. In this study, the secondary metabolites of root, stem, leaf, and flower of I. nervosa Wall. were visualized using Global Natural Products Social Molecular Networking (GNPS), MolNetEnhancer, XCMS(xcmsonline.scripps.edu) analysis, and 'ili mapping based on high performance liquid chromatography-tandem mass spectrometry (HPLC-MS/MS) data to reveal their chemical differences. Among the 11 kinds of chemical repertoires annotated by MolNetEnhancer and 16 hits against the GNPS library, 10-isobutyryloxy-8,9-epoxythymol isobutyrate (1) was revealed as the most dominant and responsible marker between the roots and the other parts. Moreover, a battery of unique MS features as well as differential markers were discovered from different parts of the plant. The chemical differences contribute to the bioactivity differences, which presented in the 2,2-diphenyl-1-picryl-hydrazyl (DPPH)assay and $\mathrm{H}_{2} \mathrm{O}_{2}$-insulted HepG2 cells and were in significant correlations with the contents of 1 . real-time reverse transcription polymerase chain reaction (RT-PCR)results demonstrated that I. nervosa Wall. extracts upregulated the mRNA expression of nuclear factor E2-related factor 2(Nrf2), heme oxygenase 1(HO-1), NAD(P)H quinone dehydrogenase 1 (NQO1), manganese superoxide dismutase (MnSOD), and glutamate-cysteine ligase catalytic subunit (GCLC) actors involved in antioxidative response in $\mathrm{H}_{2} \mathrm{O}_{2}$-challenged HepG2 cells. These findings support the roots of I. nervosa Wall. as active parts of Xiaoheiyao, and also indicate the potential antioxidant activities of other parts.
\end{abstract}

Keywords: Xiaoheiyao; GNPS molecular networking; MolNetEnhancer; XCMS online; Nrf2

\section{Introduction}

Inula nervosa Wall. is an edible and medicinal herb distributed throughout Southwest of China [1]. The roots of I. nervosa Wall. (Xiaoheiyao) are traditionally applied as culinary spice and folk medicine for treating stomachache and relieving rheumatism [2]. Phytochemical investigation and bioactivity assay on I. nervosa Wall. have mainly revealed thymols, phenylpropanoids, and diterpenes with nitric oxide and melanogenesis inhibitory activities [2-4], which dramatically differ from batch of electrophilic sesquiterpene lactones discovered from congeneric Inula species [5-7]. Furthermore, only the root of I. nervosa Wall. was approved as a new food material by the Ministry of Health of PR China in 2010 [8], whereas there is limited scientific information on the other parts and their applications.

Electrophilic natural products are the principal indirect antioxidants in culinary spices, fruits and vegetables, which are responsible for the activation of the kelch-like ECH-associated 
protein-1/nuclear factor E2-related factor 2/antioxidant response element(Keap1/Nrf2/ARE) signaling pathway, resulting in transcriptional induction of diverse cytoprotective phase 2 enzymes, including glutathione transferases (GSTs), heme oxygenase 1 (HO-1), UDP-glucuronosyltransferases (UGTs), and $\mathrm{NAD}(\mathrm{P}) \mathrm{H}$ :quinone oxidoreductase 1 (NQO1) [9-11]. In distinction with direct antioxidants, the indirect antioxidants are not consumed, with long half-lives, and are more robust to counteract the damaging effects of oxidants and electrophiles which play a role in the pathogenesis of chronic diseases, such as cancer, neurodegeneration, atherosclerosis, and non-alcoholic fatty liver disease [9]. Hence, the development of electrophilic natural products as promising antioxidants is gaining attention from pharmacologists and nutritionists.

The recently launched Global Natural Product Social (GNPS) molecular networking is an open-access knowledge base for community-wide organization and sharing of raw, processed, or identified tandem mass (MS/MS) spectrometry data [12]. Molecular networking groups MS/MS spectra based on spectral similarity, which also facilitate to dereplicate known molecules by spectral comparisons with trusted standards or community annotations, resulting in an information-rich plot of the mass spectrometry detectable chemical space [12]. In this study, the chemical space of secondary metabolites from root, stem, leaf, and flower parts of I. nervosa Wall. was visualized and annotated by GNPS molecular networking, MolNetEnhancer, XCMS((xcmsonline.scripps.edu) analysis, and 'ili mapping based on high performance liquid chromatography-tandem mass spectrometry (HPLC-MS/MS) analysis. Moreover, the antioxidant activities of different parts of I. nervosa Wall. were evaluated on the 2,2-diphenyl-1-picryl-hydrazyl (DPPH) scavenging assay and hydroperoxide $\left(\mathrm{H}_{2} \mathrm{O}_{2}\right)$-induced HepG2 cells for addressing the antioxidant difference.

\section{Materials and Methods}

\subsection{Materials}

Six batches of the whole plants of I. nervosa Wall. were collected from Liangwang Mountain, Kunming, Yunnang Province, PR China, in September 2018, and identified by Prof. Guo-Dong Li (Kunming Zhifen Biotechnology Co., Ltd.). The voucher specimens (No. XHY20180901-XHY20180906) are deposited at School of Food Science and Technology, Jiangnan University, Wuxi, Jiangsu Province, PR China. The human hepatoma carcinoma cell lines (HepG2) were obtained from the Cell Bank of the Chinese Academy of Sciences (Shanghai, China).

\subsection{Chemicals and Primary Detection Kits}

Dulbecco's modified Eagle's medium (DMEM/High glucose) was purchased from Hyclone (Logan, UT, USA), $0.25 \%$ trypsin solution with ethylene diamine tetraacetic acid(EDTA), fetal bovine serum (FBS), and penicillin streptomycin were purchased from Gibco (Grand Island, NY, USA). Detection kits of total antioxidant capacity (T-AOC), thiobarbituric acid reactive substances (TBARS), glutathione peroxidase (GSH-PX), and catalase (CAT) were purchased from Nanjing Jiancheng Bioengineering Institute (Nanjing, China). Assay kits of reaction oxygen species (ROS), cell counting kit-8 (CCK-8), and enhanced bicinchonininc acid (BCA) protein were purchased from Beyotime Biotechnology (Shanghai, China). RNA-easy Isolation Reagent kit was purchased from Vazyme (Nanjing, China). PrimeScript RT (reverse transcription) Master Mix reverse transcription kit, SYBR green PCR kit, and the q225 were purchased from Monad (Suzhou, China). DPPH, quercetin, and HPLC-grade solvents were purchased from Shanghai Aladdin Bio-Chem Technology Co., Ltd. (Shanghai, China). HPLC-grade water was obtained by filtration using a Milli-Q Direct water purification system from Millipore (Billerica, MA, USA) unless otherwise stated.

\subsection{Crude Extract Preparation of Different Parts of I. nervosa Wall}

The air-dried and powdered root, stem, leaf, and flower parts of I. nervosa Wall. (each $10 \mathrm{~g}$ ) were extracted with ethanol (1:20) three times with ultrasonic assistant for $30 \mathrm{~min}$ at $45^{\circ} \mathrm{C}$. The combined 
extracts were concentrated under reduced pressure to afford crude extracts of different parts of I. nervosa Wall. (IEs), which were further dissolved in methanol at $1.0 \mathrm{mg} / \mathrm{mL}$ and filtered by $0.2 \mu \mathrm{m}$ membrane.

\subsection{HPLC-MS/MS Analysis}

The HPLC-MS/MS analyses were developed on an HPLC system (Agilent 1200) coupled to a Thermo-Finnigan LCQ Advantage ion-trap mass spectrometer (San Jose, CA, USA) fitted with an electrospray ionization source operating in positive mode. The chromatographic separation was carried out on a Kinetex $\mathrm{C}_{18}$ column (Phenomenex; $150 \mathrm{~mm} \times 4.6 \mathrm{~mm} \times 5 \mu \mathrm{m}$ ), using an isocratic $10 \%$ acetonitrile (ACN) for 2 min and a gradient from 10\% to 99\% ACN over 20 min followed by $4 \mathrm{~min}$ washing and $4 \mathrm{~min}$ equilibrium with a flow rate of $0.6 \mathrm{~mL} / \mathrm{min}$. Both solvents contained $0.1 \%$ formic acid. The HPLC eluate was electrospray ionized at $35 \mathrm{eV}$ with capillary temperature $325^{\circ} \mathrm{C}$. An untargeted method was employed to acquire the MS/MS spectra within m/z range 100-2000.

\subsection{Molecular Networking Creation and Annotation}

A molecular network comprising MS/MS data of IEs was created using the online workflow at the GNPS website [12]. The MS/MS data were clustered with a precursor and fragment ion mass tolerance of 2.0 and 0.5 Da respectively, to create consensus spectra. The consensus spectra containing less than 2 spectra were discarded. A network was then built where edges were filtered to have a cosine score above 0.7 and more than 6 matched peaks. The consensus spectra in the network were dereplicated against spectra in GNPS' libraries which were filtered in the same threshold as the input data. Analogue search was enabled against the library with a maximum mass shift of $100 \mathrm{Da}$. The achieved GNPS molecular networking was further annotated with MolNetEnhancer [13]. The generated molecular network was imported into Cytoscape v3.7.1 (The Cytoscape Consortium, New York, NY, USA) and displayed as nodes and edges [14]. Nodes represent parent ions, and edge thickness corresponds to the cosine score between two nodes.

\subsection{Multivariate Analyses}

HPLC-MS data, including the retention time and corresponding parent mass spectra, were submitted to XCMS Online platform which was developed to process and visualize mass-spectrometrybased, complex untargeted metabolomic data [15]. For feature detection, the 'matchedFilter' method was applied, with a maximal tolerated $\mathrm{m} / \mathrm{z}$ deviation in consecutive scans of $50 \mathrm{ppm}$, minimum peak width in $10 \mathrm{~s}$, maximum peak width in $60 \mathrm{~s}$, signal/noise threshold as 6 , and minimum $\mathrm{m} / \mathrm{z}$ difference as 0.01. The 'obiwarp' method was adopted for retention time correction with step size of $1 \mathrm{~m} / \mathrm{z}$ for profile generation from the raw data files. The peaks were aligned according to bandwidth threshold as $15 \mathrm{~s}$, minimum fraction of samples as 0.5 , and width of overlapping $m / z$ slices as 0.05 . The parameters for statistics and visualization were set as default.

\section{7. 'ili Visualization}

A metadata file comprising intensity of inspected MS feature and coordinates was created and saved in comma-separated values(CSV) format. The spatial data mapping was achieved by uploading the photo of I. nervosa Wall. and metadata file [16]. A logarithmic scale was set to visualize the intensity of targeted feature in different parts of I. nervosa Wall.

\subsection{DPPH Radical Scavenging Activity Assay}

DPPH radical scavenging activity was evaluated by spectrophotometric analysis (Microplate Reader, Biotek, Winooski, VT, USA), as previously reported [17]. In brief, $2 \mathrm{~mL}$ DPPH solutions (0.1 M) were mixed with $1 \mathrm{~mL}$ samples in gradient concentrations $(0.125,0.25,0.5,1.0,2.0 \mathrm{mg} / \mathrm{mL})$ and kept at room temperature for $30 \mathrm{~min}$, then optical density values were measured at $517 \mathrm{~nm}$. 


\subsection{Intracellular Antioxidative Activity Assay}

As previously reported, the IEs were evaluated for intracellular antioxidative activities on $\mathrm{H}_{2} \mathrm{O}_{2}$-induced HepG2 cells [18]. Cells were maintained in DMEM supplemented with 10\% FBS, $100 \mathrm{U} / \mathrm{mL}$ penicillin, and $100 \mu \mathrm{g} / \mathrm{mL}$ streptomycin, in an incubator with $37{ }^{\circ} \mathrm{C}$ and humidified $5 \%$ $\mathrm{CO}_{2}$ atmosphere. Cell viabilities after treating with gradient concentrations of IEs and $\mathrm{H}_{2} \mathrm{O}_{2}$ were determined using a cell counting kit-8 (CCK-8) assay. HepG2 cells were treated with $\mathrm{H}_{2} \mathrm{O}_{2}$ at $0.1-1.6 \mathrm{mM}$ for $4 \mathrm{~h}$ and a suitable concentration was determined to induce intracellular oxidative stress. Cells were pre-cultured with IEs at $0.5,1.0$, and $2.0 \mathrm{mg} / \mathrm{mL}$ for $24 \mathrm{~h}$, then renewed with DMEM and treated with $\mathrm{H}_{2} \mathrm{O}_{2}$ for another $4 \mathrm{~h}$.

Intracellular ROS levels were measured using a $2^{\prime}, 7^{\prime}$-dichlorodihydrofluorescein diacetate (DCFH-DA) probe according to the kit instruction. Cells were washed with PBS and co-incubated with DCFH-DA for $30 \mathrm{~min}$, then observed under a fluorescence microscope (Zeiss, Jena, Germany) and fluorescence intensity was measured under a microplate reader (Biotek, Winooski, VT, USA) $\left(\lambda_{\text {excitation }}\right.$ $\left.=488 \mathrm{~nm}, \lambda_{\text {emission }}=525 \mathrm{~nm}\right)$.

The levels of TBARS, T-AOC, CAT, and GSH-PX were measured according to the kits' instructions after cells were lysed with phenylmethylsulfonyl fluoride (PMSF) dissolved radio immunoprecipitation assay (RIPA) lysis buffer in ice for $10 \mathrm{~min}$ and centrifuged at $12,000 \times \mathrm{g}$ for $5 \mathrm{~min}$ at $4{ }^{\circ} \mathrm{C}$ to afford the supernatant [19].

\subsection{Real-Time Reverse Transcription Polymerase Chain Reaction (RT-PCR)}

Total RNA was isolated from HepG2 cells using the RNA-easy Isolation Reagent kit and reverse-transcribed into cDNA using the PrimeScript RT Master Mix reverse transcription kit. The resultant cDNA was amplified by PCR using the SYBR green PCR kit and then the expression was quantified by the q225. Primers for RT-PCR are shown in Table 1. Gene expression was calculated using the $2^{-\Delta \Delta C t}$ method and normalized to $\beta$-actin.

Table 1. Primers for Real-Time Reverse Transcription Polymerase Chain Reaction (RT-PCR).

\begin{tabular}{ccc}
\hline \multirow{2}{*}{ Gene } & \multicolumn{2}{c}{ Primer $\left(\mathbf{5}^{\prime} \rightarrow \mathbf{3}^{\prime}\right)$} \\
\cline { 2 - 3 } & Gorward & Reverse \\
\hline 3-actin & TCCAGTCAGACGACACGAGCG & GCACAGAGCCTCGCCTT \\
Nrf2 & GTGGTGGAGTCGGACCTCTATG & GAATGTCTGCGCCAAAAGCTG \\
NQO1 & AAGCCAGAACAGACTCGGCAG \\
HO-1 & GAGAGGACCCATCGGA & GCCAGCAACAAAGTGCAAG \\
MnSOD & CCAGAAAATGCTATGATTGATATGAC & AAGGGAGATGTTACAGCCCAGATA \\
GCLC & AGGGAGTTTATCGCAAACCA & AAGTAACTCTGGGCATTCACA \\
\hline
\end{tabular}

\subsection{Statistical Analysis}

All data were expressed as means \pm S.E.M. (standard error of mean), obtained from at least six separate repetitions, and one representative image was selected for presentation in the Figures. Statistical analysis was performed using IBM-SPSS Inc. software (version 20.0, Armonk, NY, USA). One-way analysis of variance (ANOVA) was used to determine significant differences between means, with the significance level taken at $p<0.05$. Tukey's test was used to perform multiple comparisons between means, with the significance level $p<0.05$.

\section{Results and Discussion}

\subsection{Molecular Networking and Annotation of IEs}

Molecular networking is a visualization approach of the chemical space present in tandem MS experiments [12]. The HPLC-MS/MS spectra of IEs were grouped and constructed to create a molecular 
network based on spectral similarity (Figure 1). The molecular network exhibits an overview of the secondary metabolites and their distributions among the samples. After solvent blank removal, 412 nodes organized in 244 clusters were exhibited in the molecular network (Figure 1C). For all that, 118 parent ions were detected exclusively in root samples, 51 parent ions were found exclusively in stem samples, 52 parent ions were discovered exclusively in leaf samples, 40 parent ions were detected exclusively in flower samples, and only 11 features were shared by all of them (Figure 1D, Supporting Information Tables S1-S4). The chemical diversity of different parts of I. nervosa Wall. distinguished in the base peak chromatograms (BPCs) as well (Figure 1A), with remarkable differences of MS feature and intensity among root, stem, leaf, and flower extracts.

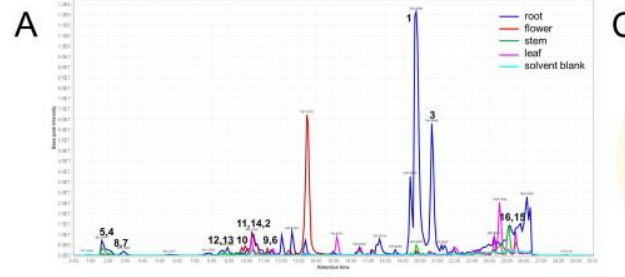

B

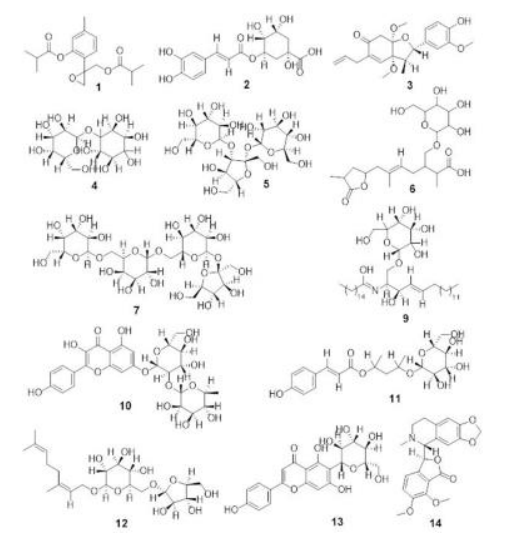

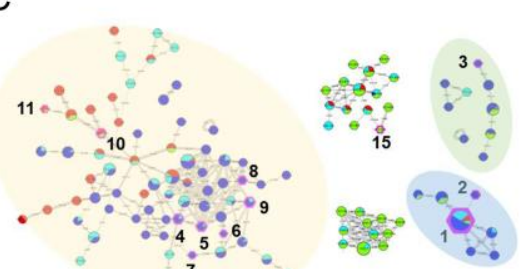
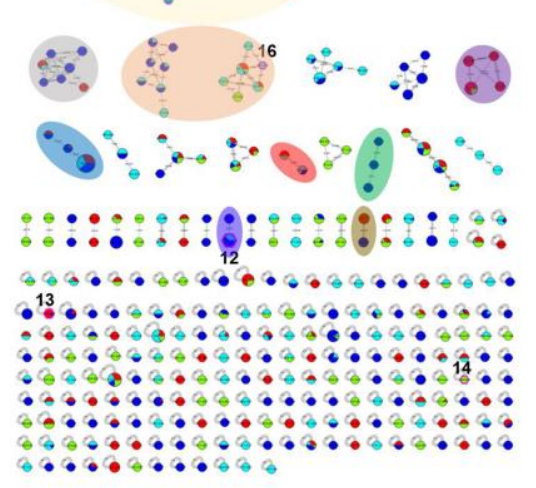

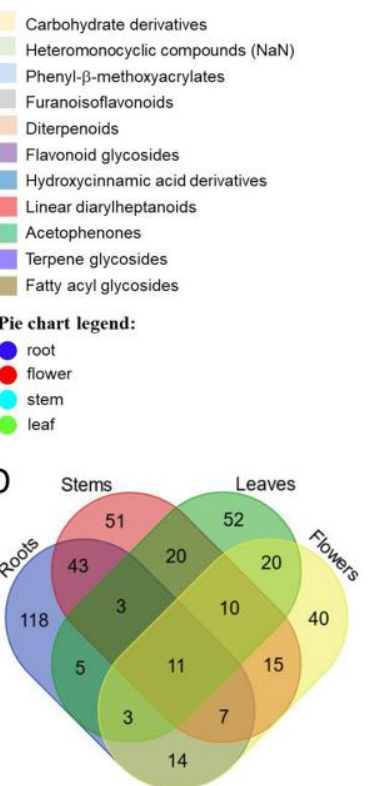

Figure 1. Chemical characterization of secondary metabolites from IEs basing on high performance liquid chromatography-tandem mass spectrometry (HPLC-MS/MS). (A) base peak chromatogram (BPC) of representative IE samples in positive mode. (B) Several chemical structures of the hits in tolerance against GNPS spectral library. (C) The molecular network of IEs [20], in which chemical classification was achieved by MolNetEnhancer at the subclass level, except for the heteromonocyclic compounds classified at the molecular framework level [21] In the molecular network, nodes represent parent ions and their sizes were designed according to the sum of precursor intensity. Nodes from solvent blanks were removed. Pie ratio was determined according to scan number of spectra. Edges represent the degree of similarity between the connected nodes and their thicknesses were corresponding to cosine scores. (D) Venn diagram with the precursor ions presented in the molecular network.

MolNetEnhancer is a workflow that combines the outputs from various in silico annotation tools and classifies chemicals through ClassyFire, which could provide a comprehensive chemical overview of metabolomic data [13,22]. As shown in Figure 1C, the molecular families were classified at the subclass level, except for the heteromonocyclic compounds. The group of heteromonocyclic family was classified at the molecular framework level since $\mathrm{NaN}$ (not-a-number) for these nodes was retrieved at the subclass level. The chemical interpretation of secondary metabolites from IEs reveals mainly 11 types of structures, including carbohydrate derivatives, heteromonocyclic compounds, phenyl- $\beta$-methoxyacrylates, furanoisoflavonoids, diterpenoids, flavonoid glycosides, hydroxycinnamic acid derivatives, linear diarylheptanoids, acetophenones, terpene glycosides, and fatty acyl glycosides.

The online dereplication against the GNPS library, the most comprehensive spectral library of natural products [23], resulted in 183 hits, which were further manually checked for the mirror spectra, cosine scores, and number of shared peaks, to yield 16 hits in tolerance (Table 2) and 
54 homologues/derivatives (Supplementary Table S5). The 16 hit compounds were empirically classified as saccharides $(4,5,7,8)$, terpenoids $(\mathbf{1}, 6, \mathbf{1 2})$, phenylpropanoids $(\mathbf{2}, \mathbf{1 1})$, flavonoids $(\mathbf{1 0}, \mathbf{1 3})$, chlorophylls $(\mathbf{1 5}, \mathbf{1 6})$, and others $(3, \mathbf{9}, \mathbf{1 4})$, which are consistent with the molecular families annotated by MolNetEnhancer. Although some thymols, phenylpropanoids, and diterpenes have already been reported [2-4], all of these hit compounds were discovered from I. nervosa Wall. for the first time. Meanwhile, the vast majority of nodes evaded all attempts to interpret them, indicating that there exists plenty of dark matter of metabolites from IEs. It is noteworthy that 10-isobutyryloxy-8,9-epoxythymol isobutyrate (1) shows as the strongest MS signal in the BPC and the biggest node in the molecular network, indicating it as the major component in the roots. Moreover, compounds 3, 6, 7, and 8 were discovered exclusively in the roots, compound $\mathbf{1 6}$ was discovered exclusively in the stems, and compounds $\mathbf{1 4}$ and $\mathbf{1 5}$ were discovered exclusively in the leaves. Some of these compositions have phytobiological effects, such as chlorophyll helping plants' photosynthesis and resveratrol protecting against pests and diseases.

Table 2. The hits in precursor tolerance against the GNPS library.

\begin{tabular}{|c|c|c|c|c|c|c|c|}
\hline Compound & $\begin{array}{l}\text { Cluster } \\
\text { Index }\end{array}$ & Adduct & $m / z$ & $\begin{array}{l}\text { Mass } \\
\text { Differ }\end{array}$ & $\begin{array}{l}\text { Cosine } \\
\text { Score }\end{array}$ & $\begin{array}{c}\text { Shared } \\
\text { Peaks }\end{array}$ & Identification \\
\hline 1 & 262 & {$[\mathrm{M}+\mathrm{H}]^{+}$} & 320.997 & 0.173 & 0.84 & 6 & $\begin{array}{l}\text { 10-Isobutyryloxy-8,9- epoxythymol } \\
\text { isobutyrate }\end{array}$ \\
\hline 2 & 357 & {$[\mathrm{M}+\mathrm{H}]^{+}$} & 354.996 & 0.004 & 0.97 & 7 & $\begin{array}{c}\text { Chlorogenic acid } \\
(2 S, 3 R, 3 \mathrm{a} S, 7 \mathrm{a} R)-5-\text { Allyl-2- } \\
\text { (4-hydroxy-3-methoxyphenyl)- }\end{array}$ \\
\hline 3 & 374 & {$\left[\mathrm{M}-\mathrm{H}_{2} \mathrm{O}+\mathrm{H}\right]^{+}$} & 357.101 & 0.068 & 0.78 & 6 & $\begin{array}{c}\text { 3a,7a-dimethoxy-3-methyl- } \\
\text { 3,3a,7,7a-tetrahydro-1- } \beta \text { enzofuran- } \\
6(2 H) \text {-one }\end{array}$ \\
\hline 4 & 414 & {$[\mathrm{M}+\mathrm{Na}]^{+}$} & 365.172 & 0.068 & 0.86 & 7 & Galactinol \\
\hline 5 & 1409 & {$[\mathrm{M}+\mathrm{Na}]^{+}$} & 527.193 & 0.035 & 0.93 & 11 & Melezitose \\
\hline 6 & 999 & {$[\mathrm{M}+\mathrm{Na}]^{+}$} & 467.250 & -1.954 & 0.81 & 6 & $\begin{array}{c}\text { (Z)-2,6-dimethyl-7- } \\
\text { (4-methyl-5-oxooxolan-2-yl)-3- } \\
\text { [[3,4,5-trihydroxy-6-(hydroxymethyl) } \\
\text { oxan-2-yl]oxymethyl]hept-5-enoic } \\
\text { acid }\end{array}$ \\
\hline 7 & 3462 & {$[\mathrm{M}+\mathrm{Na}]^{+}$} & 691.309 & 2.099 & 0.82 & 8 & Stachyose \\
\hline 8 & 6241 & {$[\mathrm{M}+\mathrm{Na}]^{+}$} & 851.262 & 0.007 & 0.8 & 17 & $\begin{array}{c}\text { Polysaccharide Hexose } \mathrm{x} 5 \\
\text { N-[(2S,3R,4E)-1- }\end{array}$ \\
\hline 9 & 3590 & {$[\mathrm{M}+\mathrm{H}]^{+}$} & 701.086 & 1.521 & 0.87 & 6 & $\begin{array}{c}\text { ( } \beta \text {-D-Galactopyranosyloxy)- } \\
\text { 3-hydroxy-4-octadecen-2-yl] } \\
\text { hexadecanamide }\end{array}$ \\
\hline 10 & 2202 & {$[\mathrm{M}+\mathrm{H}]^{+}$} & 595.011 & 0.011 & 0.97 & 6 & $\begin{array}{l}\text { Kaempferol-7-O-neohesperidoside } \\
\text { 4-[(2R,3R,4S,5S,6R)-3,4,5-trihydroxy- } \\
\text { 6-(hydroxymethyl)oxan-2-yl] }\end{array}$ \\
\hline 11 & 824 & {$[\mathrm{M}+\mathrm{Na}]^{+}$} & 432.993 & -2.170 & 0.89 & 6 & $\begin{array}{c}\text { oxypentan-2-yl } \\
(E)-3-(4-h y d r o x y p h e n y l) \\
\text { prop-2-enoate } \\
(2 R, 3 S, 4 S, 5 R, 6 R)-2-[[(2 R, 3 R, 4 R, 5 S) \text { - } \\
\text { 3,4-dihydroxy-5-(hydroxymethyl) }\end{array}$ \\
\hline 12 & 1112 & {$[\mathrm{M}+\mathrm{K}]^{+}$} & 488.030 & 0.836 & 0.87 & 8 & $\begin{array}{l}\text { oxolan-2-yl]oxymethyl]-6- } \\
{[(2 E)-3,7-\text { dimethylocta-2,6- }} \\
\text { dienoxy]oxane-3,4,5-triol }\end{array}$ \\
\hline 13 & 826 & {$[\mathrm{M}+\mathrm{H}]^{+}$} & 435.064 & 1.954 & 0.78 & 10 & Isovitexin \\
\hline 14 & 556 & {$[\mathrm{M}+\mathrm{H}]^{+}$} & 384.929 & 0.785 & 0.9 & 7 & Hydrastine \\
\hline 15 & 6464 & {$[\mathrm{M}+\mathrm{H}]^{+}$} & 871.733 & 0.143 & 0.91 & 9 & Pheophytin \\
\hline 16 & 2213 & {$[\mathrm{M}+\mathrm{H}]^{+}$} & 595.418 & 2.149 & 0.81 & 6 & Pheophorbide A \\
\hline
\end{tabular}

\subsection{Multivariate Analyses of IEs}

In order to obtain more detailed information about the metabolic profiling of IEs, the HPLC-MS data were submitted to the XCMS Online platform for a multigroup comparative analysis. Principal component analysis (PCA) conducted at the unit variance scale showed the distinction of roots with other parts and the similarity of stems with leaves in the profiling of secondary metabolites (Figure 2A). 
A batch of metabolites were responsible for the significant distinctions (Supplementary Table S6), in which compound $1(\mathrm{~m} / \mathrm{z}$ 320.9909) was found to be the most responsible marker $(p<0.01, q<$ $0.01, \mathrm{CV}<0.01)$. The concentration difference of 1 in IEs was further mapped by 'ili based on the MS intensity (Figure 2B), showing an exponential difference in the concentration of 1 with a sequence of root $>$ stem $>$ flower $>$ leaf.
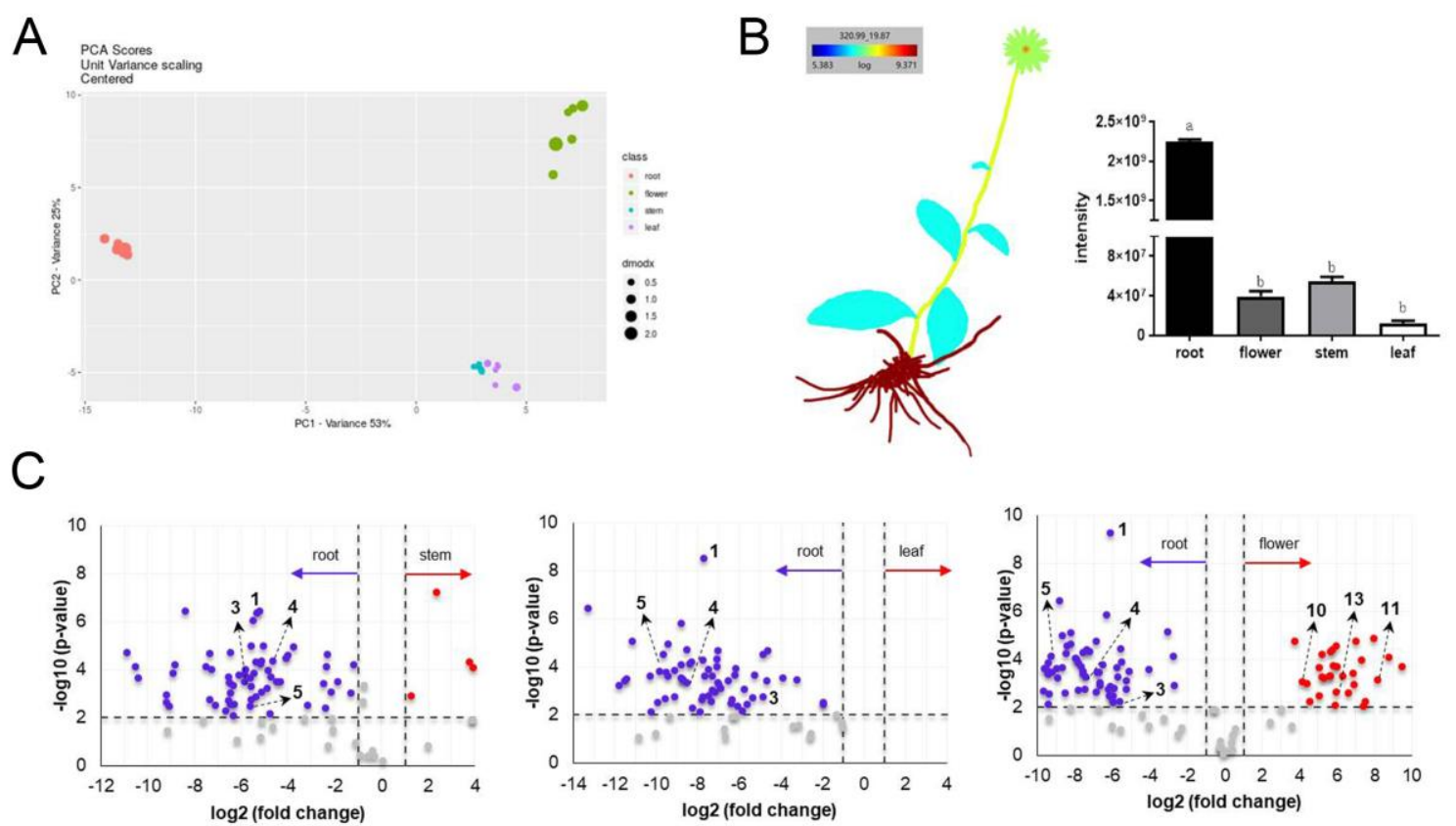

Figure 2. Multivariate analyses and visualization of metabolites from IEs. (A) PCA (Principal Component Analysis) plot of IEs based on parent ion intensity. (B) 'ili visualization of the significant feature (compound 1, $m / z$ 320.9909) intensity at the logarithmic scale $(p<0.05$ between root and other parts, $p=0.931$ between flower and stem, $p=726$ between flower and leaf, $p=0.409$ between stem and leaf). (C) Volcano plots for significant metabolite markers comparing between root with stem, leaf, and flower of $I$. nervosa Wall. (fold change $>2, p<0.01$ ).

Pairwise analyses between root with other parts were further performed to reveal their chemical differences. The significant markers were exhibited on volcano plots (fold change $>2, p<0.01$ ) (Figure 2C, Supplementary Tables S7-S9). A number of scatters were observed on the left of volcano plots, which represent the dominant features from roots, whereas much fewer dominant features (on the right) were observed from stems and leaves. Comparing the IEs of root with flower, both of them contained plenty of dominant metabolites, suggesting their great difference in secondary metabolites. Among the significant markers, compounds 1, 3, 4, and 5 dominated in root and compounds 10, 11, and 13 dominated in flower were identified via the aforementioned molecular networking. However, most of the markers are still obscure and need further chemical annotation.

The previous study on ethanol extracts of the aerial parts and underground parts of I. nervosa Wall. have revealed higher contents of total polyphenols and total flavonoids and stronger antioxidative activities from the underground parts than the aerial parts [24]. The root parts have also been reported with high content of bioactive thymol and thymyl isobutyrate [25]. Compound 1 discovered as a dominant marker in this study is a thymol derivative featured an epoxy moiety. The ring-strained epoxide is prone to undergo a Michael addition with nucleophilic sites, such as cysteine, serine, lysine residues in proteins, DNA, and glutathione, interfering with a variety of biological functions [11]. Therefore, compound 1 might play a role in the bioactivity and traditional application of Xiaoheiyao. Meanwhile, as supporting from the chemical markers 3, 10, 11, and 13, the chemical constituents of polyphenols and flavonoids from IEs may vary in different parts. Moreover, for a comprehensive chemical comparison between different parts of $I$. nervosa Wall., the residue 
markers, such as the significant feature of $\mathrm{m} / \mathrm{z} 787.2664$ at $13.56 \mathrm{~min}$ (Figures 1A and 2C), deserve further chemical identification.

\subsection{DPPH Radical Scavenging Activity}

Dietary antioxidants conduct a one-electron reaction with free radicals in vitro. DPPH has been widely applied for the determination of in vitro antioxidant activity of plant extracts and pure compounds due to its relatively stability.

The DPPH scavenging activities of IEs were evaluated and compared in Figure 3. The IEs scavenged the DPPH radical in a dose-dependent manner at concentrations of $0.125-2.0 \mathrm{mg} / \mathrm{mL}$, and DPPH radicals were almost quenched by the root and flower IEs at $1.0 \mathrm{mg} / \mathrm{mL}$. Quercetin with an half maximal inhibitory concentration $\left(\mathrm{IC}_{50}\right.$ ) value of $3.47 \pm 0.10 \mu \mathrm{g} / \mathrm{mL}$ was applied as a positive control. The root and flower IEs exhibited similar $\mathrm{IC}_{50}$ values of DPPH scavenging activity at $0.218 \pm$ 0.04 and $0.284 \pm 0.01 \mathrm{mg} / \mathrm{mL}$, whereas the stem and leaf IEs owned $\mathrm{IC}_{50}$ values at $0.479 \pm 0.03$ and 1.055 $\pm 0.05 \mathrm{mg} / \mathrm{mL}$, respectively. The DPPH scavenging activities of IEs are in descending order as root > flower $>$ stem $>$ leaf. The extracts from underground parts of I. nervosa Wall. have stronger radical scavenging activities than the aerial parts, which might owe to higher content of total polyphenols and total flavonoids, as in a previous report [24].

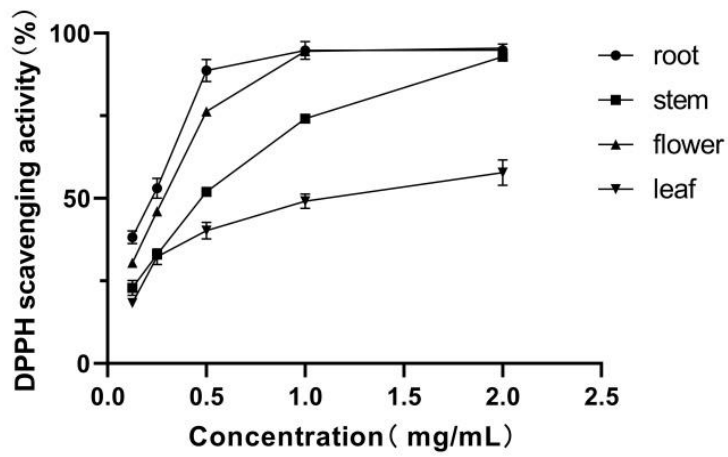

Figure 3. DPPH radical scavenging activities of IEs. Each value is expressed as means \pm S.E.M. $(n=6)$.

\subsection{Intracellular Antioxidative Activities of IEs}

Hydroperoxide is one of the most abundantly endogenic oxidizers inducing intracellular oxidative stress. Enzymatic removal of non-radical electrophiles in two-electron redox reactions, such as CAT and GSH-PX, neutralizing $\mathrm{H}_{2} \mathrm{O}_{2}$ into water and oxygen, is considered as the major intracellular anti-oxidative mechanism [26,27].

In the present study, $\mathrm{H}_{2} \mathrm{O}_{2}$-induced HepG2 cells were used to evaluate the intracellular anti-oxidative activities of IEs. When the HepG2 cells were treated with $1.2 \mathrm{mM} \mathrm{H}_{2} \mathrm{O}_{2}$ for $4 \mathrm{~h}$, the cell viability was decreased to $55.94 \%(p<0.05)$ relative to control and ROS production increased to $200.97 \%(p<0.05)$ relative to control. The intracellular total antioxidant capability and the enzyme activities to eliminate $\mathrm{H}_{2} \mathrm{O}_{2}$ was significantly lower than the control, while the TBARS level was a fold increase over the control (Figure 4).

The IEs were initially confirmed with no significant cytotoxic effect on HepG2 cells at the inspected concentration of $0.5-2.0 \mu \mathrm{g} / \mathrm{mL}$ in the CCK-8 assay. Pretreating with IEs, the viabilities of $\mathrm{H}_{2} \mathrm{O}_{2}$-insulted HepG2 cells were enhanced in a dose-dependent manner. All the IEs increased the cell viabilities in a sequence of root $>$ flower $>$ stem $>$ leaf. The IEs at all assayed concentrations, especially at $2.0 \mu \mathrm{g} / \mathrm{mL}$, were effective in reducing ROS and TBARS levels, restoring T-AOC, CAT, and GSH-PX activities in the cells that were affected due to $\mathrm{H}_{2} \mathrm{O}_{2}$, and showed with gradient changes as the concentration changed (Figure 4). The root and flower IEs exhibited relatively lower ROS and TBARS levels, whereas significantly higher CAT and GSH-PX activities than those of stem and leaf $(p<0.05)$. Taken together, 
the root and flower of I. nervosa Wall. possess distinguished antioxidant capacity from the stems and leaves.

A

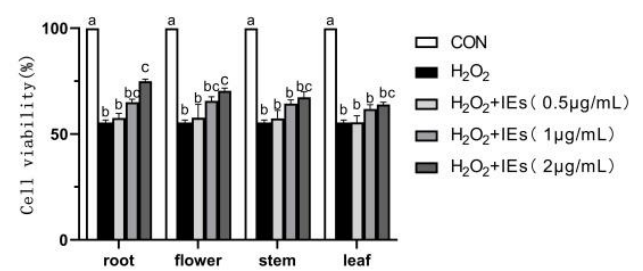

C

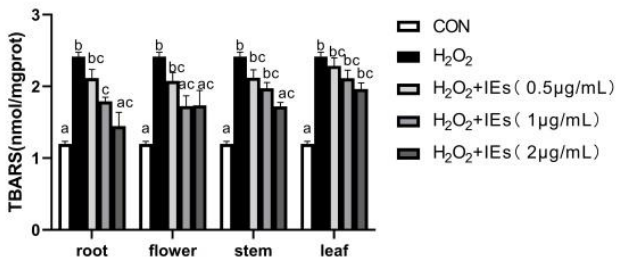

$\mathbf{E}$

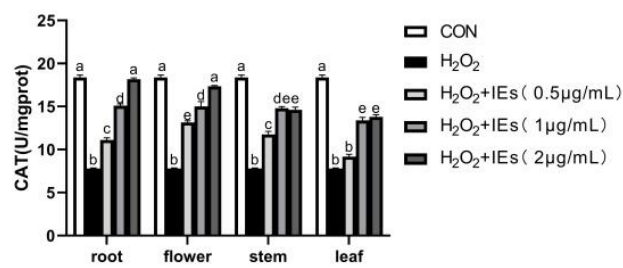

B

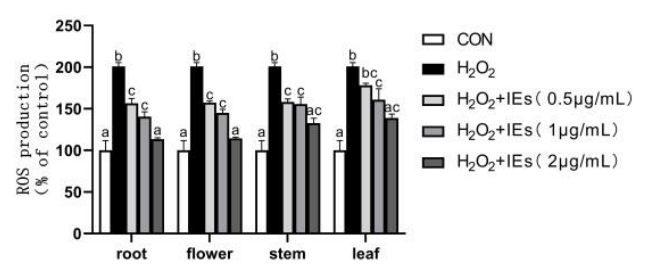

D

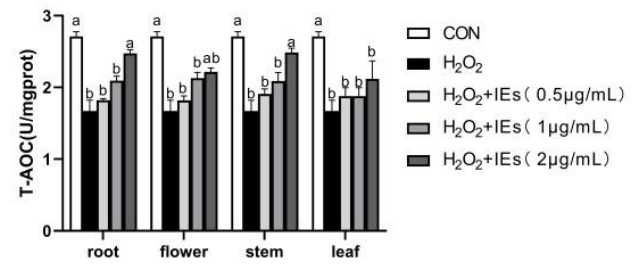

F

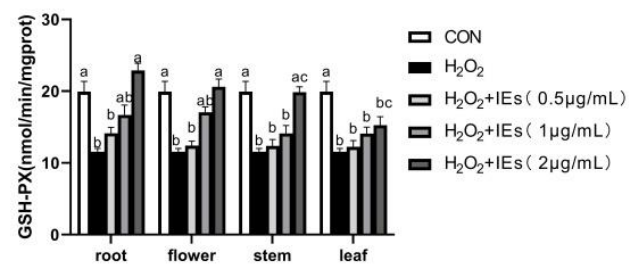

Figure 4. Effects of IEs on (A) cell viability, (B) ROS production, (C) TBARS level, (D) T-AOC level, (E) CAT, and (F) GSH-PX activities in $\mathrm{H}_{2} \mathrm{O}_{2}$-induced HepG2 cells. Values were presented as mean \pm S.E.M. $(n=8)$. Bars within a figure without a common superscript differ significantly at $p<0.05$.

Some early investigators hold the idea of a possible homeostatic equilibrium, in which the nutritional antioxidants would cause a decrease in endogenous antioxidant protection through feedback inhibition of regulatory mechanisms by increasing exogenous antioxidant capacity [26]. Instead, investigations on nutritional antioxidants, such as phenolic compounds, isothiocyanates, and other phytochemicals, actually observed an increased endogenous antioxidant protection, which was achieved by activating signal transduction pathways leading to altered gene expression, particularly in the Keap1/Nrf2/ARE pathway [26]. It is noteworthy that the root and flower IEs exhibited both the DPPH scavenging activities and increased intracellular antioxidant protection, indicating that the IEs would activate intracellular antioxidant signal pathways.

\subsection{Effects of IEs on Nrf2/ARE Pathway Activation}

$\mathrm{Nrf2}$ is the main redox-sensitive transcription factor upregulating the expression of cell-protective genes in the oxidative stress response [28]. Once the Nrf2 signaling pathway is activated, Nrf2 is dissociated from Keap1-Nrf2 complex in the cytoplasm and transferred to the nucleus, where it binds to AREs in the promoter regions. Then, a series of detoxifying and antioxidant defense genes, such as GSTs, HO-1, NQO1, and MnSOD, are subsequently expressed to play anti-oxidative, anti-inflammatory, and anti-apoptotic roles [29]. GSH is a universal non-protein intracellular thiol present in millimolar concentrations, participating in the antioxidant process either directly by detoxifying ROS or indirectly via GSH-PX catalyzed reactions [30]. Glutamylcysteine synthase (GCL), which comprises of the catalytic subunit (GCLC) and the modulating subunit, is the rate-limiting enzyme in the GSH synthesis pathway [31]. 
In this context, the effects of IEs on the Nrf2/ARE pathway were examined via RT-PCR of Nrf2, HO-1, NQO1, MnSOD, and GCLC mRNA expression (Figure 5). All of the IEs relieved the impairment induced by $\mathrm{H}_{2} \mathrm{O}_{2}$, improving the Nrf2 and downstream genes' HO-1, NQO1, MnSOD, and GCLC mRNA expression in a dose-dependent manner. The Nrf2 activation effect is relatively stronger in the root IE than in flower, stem, and leaf IEs at the concentration of $2.0 \mu \mathrm{g} / \mathrm{mL}$. These results emphasized that the IEs can activate the Nrf2/ARE pathway to act as indirect antioxidants.

A

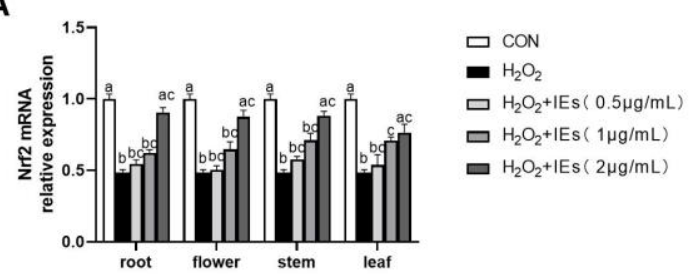

C

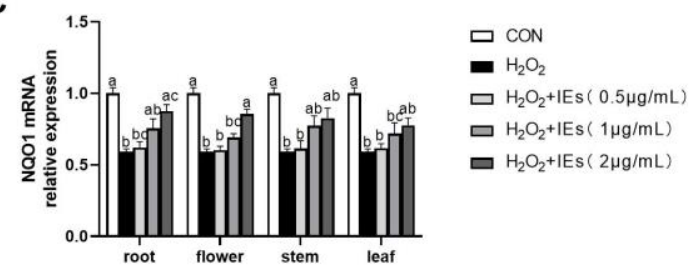

E

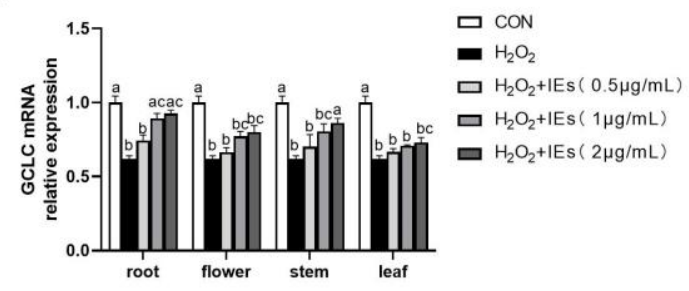

B

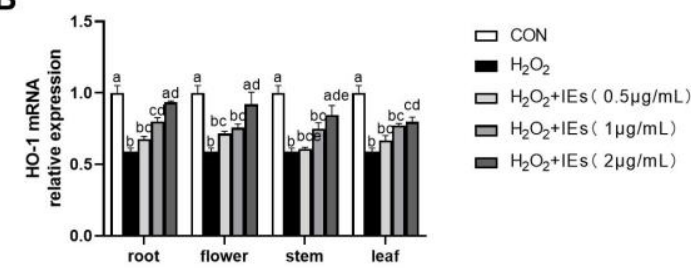

D

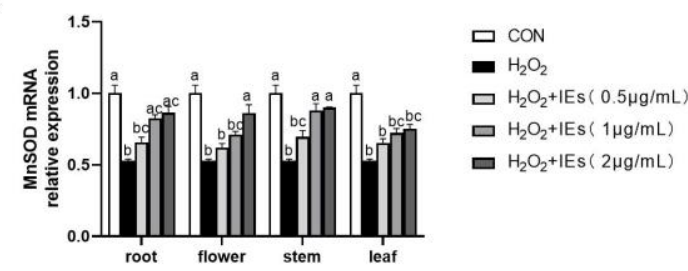

Figure 5. Effects of IEs on the mRNA expression of (A) Nrf2, (B) HO-1, (C) NQO1, (D) MnSOD, and (E) GCLC in $\mathrm{H}_{2} \mathrm{O}_{2}$-induced HepG2 cells. Values were presented as mean \pm S.E.M. $(n=6)$. Bars within a figure without a common superscript differ significantly at $p<0.05$.

\subsection{Correlations between Compound 1 and Antioxidant Parameters}

The chemical difference among IEs is responsible for their antioxidative activity variety. Compound 1 was illustrated as the most abundant and significant feature from the root extract. Herein, the correlations between compound $\mathbf{1}$ and antioxidant parameters were analyzed to reveal the relationships. As shown in Figure 6, the content of $\mathbf{1}$ has significantly positive correlations with DPPH, GSH-PX, and CAT activities $(p<0.01)$ and significantly negative correlation with ROS and TBARS levels $(p<0.05)$. 

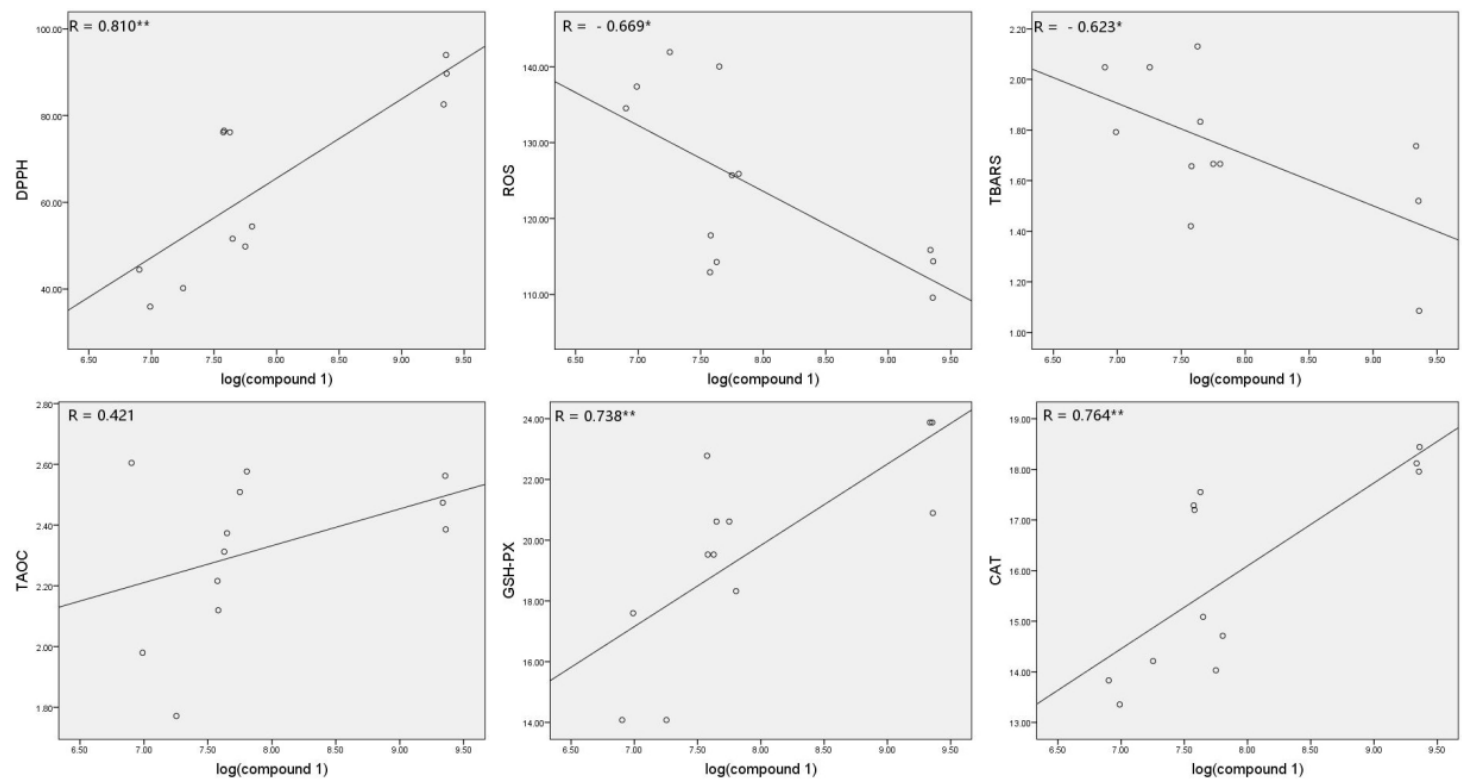

Figure 6. Correlations between the content of compound 1 (ion intensity at logarithmic scale) and antioxidant parameters $\left({ }^{*} p<0.05 ;{ }^{* *} p<0.01\right)$.

Compound $\mathbf{1}$ is a thymol $d i$-isobutyrate without active phenolic hydroxyl group or proton to diminish DPPH radicals through a hydrogen atom transfer mechanism [32], a single electron transfer mechanism [33], or a sequential proton loss electron transfer mechanism [34,35]. Hence, the DPPH scavenging activity of compound $\mathbf{1}$ deserves further confirmation after its purification.

As mentioned earlier, the epoxy moiety of $\mathbf{1}$ could carry out a ring-opening and alkylation of one or more cysteine sulfhydryl groups of Keap1, leading to Nrf2 nuclear accumulation and upregulation of the expression of a large array of cytoprotective enzymes in response to oxidative assault. Human Keap 1 contains 27 cysteines, some of which are purported to be the targets of electrophiles [36,37]. Cys151 has been reported as the most reactive site towards electrophilic natural products xanthohumol, isoliquiritigenin, and 10-shogaol [38]. The positive correlations between the content of $\mathbf{1}$ and GSH-PX and CAT activities indicate that compound $\mathbf{1}$ has a potential intracellular antioxidative effect which might be achieved by Nrf2 activation.

\section{Conclusions}

In summary, this study demonstrated that the application of comprehensive cheminformatics tools could facilitate the heterogeneity revelation in food chemistry and biological activities. By comparing the chemical space and antioxidative activities of ethanol extracts from root, stem, leaf, and flower parts of I. nervosa Wall., 10-isobutyryloxy-8,9-epoxythymol isobutyrate (1) was revealed as the most dominant and responsible marker from the root parts, that is, drawn from GNPS molecular networking, MolNetEnhancer, XCMS analysis, and 'ili mapping. The direct and indirect antioxidative effects of IEs were found to correlate with the content of $\mathbf{1}$. Moreover, the IEs were deduced to activate the Nrf2/ARE pathway to serve as indirect antioxidants. These findings also support the traditional application of the roots of I. nervosa Wall. as Xiaoheiyao and indicate $\mathbf{1}$ as one of the major functional factors, and it could be seen as a quality control factor as well. The results also cue the potential antioxidant of other parts, especially the flowers, and its toxicities need to be investigated before its application could be further studied. 
Supplementary Materials: The supporting information is available on the online version. Table S1. The unique features from the root IE, Table S2. The unique features from the stem IE, Table S3. The unique features from the leaf IE, Table S4. The unique features from the flower IE, Table S5. The analog hits against GNPS library, Table S6. List of differential secondary metabolites from root, stem, leaf, and flower IEs, Table S7. List of differential secondary metabolites from root and stem IEs, Table S8. List of differential secondary metabolites from root and leaf IEs, Table S9. List of differential secondary metabolites from root and flower IEs.

Author Contributions: Conceptualization, X.-r.C.; methodology, W.-1.D. and W.Z.; validation, X.-r.C., W.Z., W.-1.D. and G.-w.L.; formal analysis, W.Z. and W.-1.D.; investigation, W.Z.; resources, G.-w.L.; data curation, W.Z. and W.-l.D.; writing — original draft preparation, W.Z.; writing-review and editing, X.-r.C. and W.Z.; visualization, X.-r.C.; supervision, G.-w.L.; project administration, X.-r.C.; funding acquisition, X.-r.C. All authors have read and agreed to the published version of the manuscript.

Funding: This research was funded by the National Natural Science Foundation of China (31700301).

Conflicts of Interest: The authors declare that they have no conflict of interest.

\section{References}

1. Zhao, L.; Xin, L.Q.; Li, Y.Q. Research advance of Inula nervosa Wall. in China. Food Drug 2007, 9, 56-57.

2. Yan, L.; Cheng, X.R.; Zeng, Q.; Qin, J.J.; Zhang, W.D.; Jin, H.Z. Phytane and neoclerodane diterpenes from the aerial parts of Inula nervosa Wall. Biochem. Syst. Ecol. 2001, 39, 700-703. [CrossRef]

3. Yan, L.; Huang, Y.; Fu, J.J.; Qin, J.J.; Zeng, Q.; Zhu, Y.; Yan, S.K.; Zhang, W.D.; Jin, H.Z. Three new phenylpropanoids from Inula nervosa Wall. Helv. Chim. Acta 2010, 93, 1418-1421. [CrossRef]

4. Fujita, H.; Motokawa, T.; Katagiri, T.; Yokota, S.; Yamamoto, A.; Himeno, M.; Tanaka, Y. Inulavosin, a melanogenesis inhibitor, leads to mistargeting of tyrosinase to lysosomes and accelerates its degradation. J. Invest. Dermatol. 2009, 129, 1489-1499. [CrossRef]

5. Cheng, X.R.; Zeng, Q.; Ren, J.; Qin, J.J.; Zhang, S.D.; Shen, Y.H.; Zhu, J.X.; Zhang, F.; Chang, R.J.; Zhu, Y.; et al. Sesquiterpene lactones from Inula falconeri, a plant endemic to the Himalayas, as potential anti-inflammatory agents. Eur. J. Med. Chem. 2011, 46, 5408-5415. [CrossRef]

6. Cheng, X.R.; Zhang, S.D.; Wang, C.H.; Ren, J.; Qin, J.J.; Tang, X.; Shen, Y.H.; Yan, S.K.; Jin, H.Z.; Zhang, W.D. Bioactive eudesmane and germacrane derivatives from Inula wissmanniana Hand.-Mazz. Phytochemistry 2013, 96, 214-222. [CrossRef]

7. Wang, G.W.; Qin, J.J.; Cheng, X.R.; Shen, Y.H.; Shan, L.; Jin, H.Z.; Zhang, W.D. Inula sesquiterpenoids: Structural diversity, cytotoxicity and anti-tumor activity. Expert Opin. Inv. Drug 2014, 23, 317-345. [CrossRef]

8. Ministry of Health of PR China. Announcement of the Ministry of Health of PR China No.9 of 2010. Chin. J. Food Hyg. 2010, 22, 380.

9. Dinkova-Kostova, A.T.; Talalay, P. Direct and indirect antioxidant properties of inducers of cytoprotective proteins. Mol. Nutr. Food Res. 2008, 52, S128-S138. [CrossRef]

10. Ahn, Y.H.; Liu, H.; Wang, X.J.; Zhang, Y.; Stephenson, K.K.; Boronina, T.N.; Cole, R.N.; Dinkova-Kostova, A.T.; Talalay, P.; Cole, P.A. Electrophilic tuning of the chemoprotective natural product sulforaphane. Proc. Natl. Acad. Sci. USA 2010, 102, 9590-9595. [CrossRef]

11. Gersch, M.; Kreuzer, J.; Sieber, S.A. Electrophilic natural products and their biological targets. Nat. Prod. Rep. 2012, 29, 659-682. [CrossRef] [PubMed]

12. Wang, M.; Carver, J.J.; Phelan, V.V.; Sanchez, L.M.; Garg, N.; Peng, Y.; Nguyen, D.D.; Watrous, J.; Kapono, C.A.; Luzzatto-Knaan, T.; et al. Sharing and community curation of mass spectrometry data with Global Natural Products Social Molecular Networking. Nat. Biotechnol. 2016, 34, 828-837. [CrossRef] [PubMed]

13. Ernst, M.; Kang, K.B.; Caraballo-Rodriguez, A.M.; Nothias, L.F.; Wandy, J.; Chen, C.; Wang, M.; Rogers, S.; Medema, M.H.; Dorrestein, P.C.; et al. MolNetEnhancer: Enhanced molecular networks by integrating metabolome mining and annotation tools. Metabolites 2019, 9, 144. [CrossRef]

14. Shannon, P.; Markiel, A.; Ozier, O.; Baliga, N.S.; Wang, J.T.; Ramage, D.; Amin, N.; Schwikowski, B.; Ideker, T. Cytoscape: A software environment for integrated models of biomolecular interaction networks. Genome Res. 2003, 13, 2498-2504. [CrossRef] [PubMed]

15. Gowda, H.; Ivanisevic, J.; Johnson, C.H.; Kurczy, M.E.; Benton, H.P.; Rinehart, D.; Nguyen, T.; Ray, J.; Kuehl, J.; Arevalo, B.; et al. Interactive XCMS Online: Simplifying advanced metabolomic data processing and subsequent statistical analyses. Anal. Chem. 2014, 86, 6931-6939. [CrossRef] [PubMed] 
16. Protsyuk, I.; Melnik, A.V.; Nothias, L.F.; Rappez, L.; Phapale, P.; Aksenov, A.A.; Bouslimani, A.; Ryazanov, S.; Dorrestein, P.C.; Alexandrov, T. 3D molecular cartography using LC-MS facilitated by Optimus and 'ili software. Nat. Protoc. 2017, 13, 134-154. [CrossRef] [PubMed]

17. Rosero, J.C.; Cruz, S.; Osorio, C.; Hurtado, N. Analysis of phenolic composition of byproducts (seeds and peels) of Avocado (Persea americana Mill.) cultivated in Colombia. Molecules 2019, 24, 3209. [CrossRef]

18. Salla, S.; Sunkara, R.; Ogutu, S.; Walker, L.T.; Verghese, M. Antioxidant activity of papaya seed extracts against $\mathrm{H}_{2} \mathrm{O}_{2}$ induced oxidative stress in HepG2 cells. LWT-Food Sci. Technol. 2016, 66, 293-297. [CrossRef]

19. Sohn, S.H.; Kim, S.K.; Kim, Y.O.; Kim, H.D.; Shin, Y.S.; Yang, S.O.; Kim, S.Y.; Lee, S.W. A comparison of antioxidant activity of Korean White and Red Ginsengs on H2O2-induced oxidative stress in HepG2 hepatoma cells. J. Ginseng Res. 2013, 37, 442-450. [CrossRef]

20. Available online: https://gnps.ucsd.edu/ProteoSAFe/status.jsp?task=74fb92bf0553404ab0ffdc5376cfc44c (accessed on 26 April 2019).

21. Available online: https://gnps.ucsd.edu/ProteoSAFe/status.jsp?task=a26a132027a242fc993df75766ec784d (accessed on 6 October 2019).

22. Feunang, Y.D.; Eisner, R.; Knox, C.; Chepelev, L.; Hastings, J.; Owen, G.; Fahy, E.; Steinbeck, C.; Subramanian, S.; Bolton, E.; et al. ClassyFire: Automated chemical classification with a comprehensive, computable taxonomy. J. Cheminform. 2016, 8, 61. [CrossRef]

23. Mohimani, H.; Gurevich, A.; Shlemov, A.; Mikheenko, A.; Korobeynikov, A.; Cao, L.; Shcherbin, E.; Nothias, L.F.; Dorrestein, P.C.; Pevzner, P.A. Dereplication of microbial metabolites through database search of mass spectra. Nat. Commun. 2018, 9, 4035. [CrossRef] [PubMed]

24. He, A.N.; She, C.W.; Zeng, J.Y.; Peng, S.X. Comparison study on in vitro and in vivo antioxidant activities of Inula nervosa Wall. extracts from different parts. Chin. Pharmacol. Bull. 2016, 32, 79-83.

25. Li, K.; Shi, L.Z.; Chen, D.; Hu, Y.B.; Lu, M.F.; Li, R.C.; Li, S.X. Content determination of thymol and thymyl isobutyrate in Inula nervosa Wall from different parts, different habitats and different harvest periods by HPLC. J. Hunan Univ. Chin. Med. 2013, 33, 41-44.

26. Forman, H.J.; Davies, K.J.; Ursini, F. How do nutritional antioxidants really work: Nucleophilic tone and para-hormesis versus free radical scavenging in vivo. Free Radic. Biol. Med. 2014, 66, 24-35. [CrossRef]

27. Joko, S.; Watanabe, M.; Fuda, H.; Takeda, S.; Furukawa, T.; Hui, S.-P.; Shrestha, R.; Chiba, H. Comparison of chemical structures and cytoprotection abilities between direct and indirect antioxidants. J. Funct. Foods 2017, 35, 245-255. [CrossRef]

28. Jaiswal, A.K. Nrf2 signaling in coordinated activation of antioxidant gene expression. Free Radicals Biol. Med. 2004, 36, 1199-1207. [CrossRef] [PubMed]

29. Lee, C. Collaborative power of Nrf2 and PPAR $\gamma$ activators against metabolic and drug-induced oxidative injury. Oxid. Med. Cell. Longev. 2017, 2017, 1378175. [CrossRef]

30. Teskey, G.; Abrahem, R.; Cao, R.; Gyurjian, K.; Islamoglu, H.; Lucero, M.; Martinez, A.; Paredes, E.; Salaiz, O.; Robinson, B.; et al. Chapter five-Glutathione as a marker for human disease. Adv. Clin. Chem. 2018, 87, 141-159.

31. Gipp, J.J.; Chang, C.; Mulcahy, R.T. Cloning and nucleotide sequence of a full-length cDNA for human liver gamma-glutamylcysteine synthetase. Biochem. Biophys. Res. Commun. 1992, 185, 29-35. [CrossRef]

32. Litwinienko, G.; Ingold, K.U. Solvent effects on the rates and mechanisms of reaction of phenols with free radicals. Acc. Chem. Rev. 2007, 40, 222-230. [CrossRef]

33. Galian, R.E.; Litwinienko, G.; Perez-Prieto, J.; Ingold, K.U. Kinetic solvent effects on the reaction of an aromatic ketone $\pi, \pi^{*}$ triplet with phenol. Rate-retarding and rate-accelerating effects of hydrogen-bond acceptor solvents. J. Am. Chem. Soc. 2007, 129, 9280-9281. [CrossRef] [PubMed]

34. Litwinienko, G.; Ingold, K.U. Abnormal solvent effects on hydrogen atom abstraction. 2. Resolution of the curcumin antioxidant controversy. The role of sequential proton loss electron transfer. J. Org. Chem. 2004, 69, 5888-5896. [CrossRef] [PubMed]

35. Litwinienko, G.; Ingold, K.U. Abnormal solvent effects on hydrogen atom abstraction. 3. Novel kinetics in sequential proton loss electron transfer chemistry. J. Org. Chem. 2005, 70, 8982-8990. [CrossRef]

36. Eggler, A.L.; Liu, G.; Pezzuto, J.M.; van Breemen, R.B.; Mesecar, A.D. Modifying specific cysteines of the electrophile-sensing human Keap1 protein is insufficient to disrupt binding to the Nrf2 domain Neh2. Proc. Natl. Acad. Sci. USA 2005, 102, 10070-10075. [CrossRef] [PubMed] 
37. Holland, R.; Hawkins, A.E.; Eggler, A.L.; Mesecar, A.D.; Fabris, D.; Fishbein, J.C. Prospective type 1 and type 2 disulfides of Keap1 protein. Chem. Res. Toxicol. 2008, 21, 2051-2060. [CrossRef]

38. Luo, Y.; Eggler, A.L.; Liu, D.; Liu, G.; Mesecar, A.D.; van Breemen, R.B. Sites of alkylation of human Keap1 by natural chemoprevention agents. J. Am. Soc. Mass Spectrom. 2007, 18, 2226-2232. [CrossRef]

Sample Availability: Samples of the compounds are not available from the authors.

Publisher's Note: MDPI stays neutral with regard to jurisdictional claims in published maps and institutional affiliations.

(C) 2020 by the authors. Licensee MDPI, Basel, Switzerland. This article is an open access article distributed under the terms and conditions of the Creative Commons Attribution (CC BY) license (http://creativecommons.org/licenses/by/4.0/). 